\title{
Improvement of patch selection in exemplar-based image inpainting
}

\author{
Qian Fan ${ }^{\mathrm{a}^{*}}$, Lifeng Zhang ${ }^{*}$ \\ *Department of Electrical Engineering and Electronics \\ Kyushu Institute of Technology \\ Sensui-cho 1-1, Tobata-ku, Kitakyushu-shi, 804-0015, Fukuoka, Japan \\ aEmail:cnzshakka@gmail.com
}

\begin{abstract}
In the existing exemplar based image inpainting algorithms, the most similar match patches are used to inpaint the destroyed region, and they are searched in the whole source region in a fixed size. However, sometimes it would decrease the connectivity of structure and clearness of texture while increases the time complexity of this algorithm. To solve these problems, firstly we proposed an adaptive sample algorithm based on patch sparsity, it calculates the patch sparsity which divided the patches' location into three types (smooth type, transition type and edge type). Then the size of the sample patch can be adaptively changed according to the type. Secondly it proposed a candidate patch system to improve the patch matching rate. From the result, we can see that the proposed method can match more significant patches than the traditional method, and it can give a better texture inpainting effect, especially when processing the complex and regular textures in the image which has a large destroyed region.
\end{abstract}

Keywords: image inpainting, exemplar-based, adaptive sample, candidate patch system.

\section{Introduction}

The concept of digital image inpainting is firstly mentioned by Bertalmio ${ }^{(1)}$ in 2000 , for many reasons, one image may be destroyed by human, nature and other factors, some regions in the image may lost its information. Digital image inpainting technology is by using the remaining part of the image to maintain the integrity of the original image and now it is becoming a very important topic and comes out a lot of applications of preserving ancient painting, movie processing, image coding and transmission.

The most fundamental in-painting approaches are inpainting the image at pixel level, they diffuse the pixel information from the remaining regions into the missing regions ${ }^{(1-4)}$. These algorithms are well founded on the theories of partial differential equation (PDE) and variational method. Bertalmio et al. ${ }^{(1)}$ filled in holes by continuously propagating the isophote (i.e., lines of equal gray values) into the missing region. To improve the result, they further introduced the Navier-Strokes equation in fluid dynamics and applied it into the inpainting processing ${ }^{(2)}$. Afonso proposed a new fast algorithm firstly solving the constrained optimization formulation of regularized image restoration.. The approach can be used with any type of convex regularizers and it is based upon a VS technique ${ }^{(5)}$. Zhu improved the classical PDE model and proposed a novel PDE framework for the striped texture image inpainting, it employed the orientation field to diffuse the gray information along a significant direction ${ }^{(6)}$. The proposed method ${ }^{(7)}$ overcome the disadvantage of high calculation load made by the traditional PDE method and improve the inpainting performance by proposing an enhanced Curvature-Driven Diffusions (ECDD) model and a fast local non-texture in painting scheme. FMM algorithm is also one research hotspot of pixel level algorithms. It firstly measures the direction, the distance, and the level set influences made by the neighbor pixels, then through them calculating the pixel's gray value. Telea firstly introduced this algorithm and gave the basic assumption of it. It inpaints the image very quickly and has a similar result in the visual effect ${ }^{(8)}$. Yang tries to employ the anisotropic diffusion theory into the FMM algorithm and have a good result in large region in-painting ${ }^{(9)}$. In the improved method $^{(10)}$, the author changed the traditional direction index and gave a new direction selection method, it can select more significant pixels and enhance the edge effect. These methods have successfully achieved to maintain the integrity of the image, and can generate excellent results for 
the missing region which has non-textured or smaller relationship with the image. However, this category of approaches tends to produce the smooth effect in the textured region and larger missing region.

The second category approaches are the exemplar-based inpainting algorithm. These approaches propagate the image information from the known region into the missing region at the patch level. This idea is firstly proposed by Criminisi in $2006^{(11)}$. But it stems from the texture synthesis technique proposed in ${ }^{(12)}$. It samples the most suitable patch from the known region to synthesize the texture. However, natural images are composed of structures and textures, in which the structures always constitute one image's primal sketches (e.g., the edges, corners, etc.) while the textures are always represented the image regions with homogenous patterns or feature statistics (including the flat patterns). So only by texture synthesis technique it cannot clearly repair the missing region with composite textures and structures. To take the advantages of both diffusion-based method and texture synthesis technique, Bertalmio et al. ${ }^{(13)}$ firstly divided the image into structure and texture layers, then using diffusion-based method to in-paint the structure layer and texture synthesis technique to in-paint texture layer. By this processing, the smooth effect disadvantage brought from the diffusion-based in-painting algorithm is well overcome, but it is still very hard to recover the larger missing structures.Criminisi et al. ${ }^{(11)}$ designed an exemplar-based in-painting algorithm by propagating the known patches (i.e., exemplars) into the missing patches gradually. To handle the missing region with composite textures and structures, patch priority is defined to encourage the filling-in of patches on the structure. In the proposed $\operatorname{method}^{(14)}$, Zoran performed a better result by learning a better patch priors. It firstly presented a generic, simple Gaussian Mixture prior, and then applying it on a generic framework for the whole image restoration. The proposed $^{(15)}$ introduced three basic techniques: copy-and-paste texture synthesis, geometric partial differential equations (PDEs), and coherence among neighboring pixels. It combined these three building blocks in a variational model and completed to approximate the minimum of the proposed energy functional.To improve the result in a more natural quality with high performance, Chung introduced the minimum error boundary of cut technique into the exemplar-based method ${ }^{(16)} . \mathrm{Li}^{(17)}$ designed an adaptively size-changed patch according to the location, which can generate a better result in inpainting the region with complex texture. In the method ${ }^{(18)}$,the fast priority and the coherent nearest neighbor search method were employed to produces better completeness of linear edges and reduces the error propagation problem. Compared with diffusion-based in-painting algorithms, the exemplar-based in-painting algorithms has an improvement in inpainting the image which has complex texture, especially has a good result in inpainting the large missing region.

This paper learns from the traditional method ${ }^{(11)}$ and try to inpaint the image on patch level. And it tries to overcome the most important disadvantage of the traditional method: the size of the sample and the selection of good exemplars. It is organized as follows. In section 2, it introduced the theory of the traditional exemplar-based method. In section 3 , it proposed two improvements to give a better result. Contains the detail of the adaptive sample size algorithm and the candidate patch system. And the experiments and comparisons with the traditional methods are performed in the section 4. At last, we conclude the work in the section 5 .

\section{Theory of the exemplar-based method}

The main idea of the exemplar-based method is an isophote-driven image sampling process. Figure 1 shows the theory of the exemplar-based inpainting method.
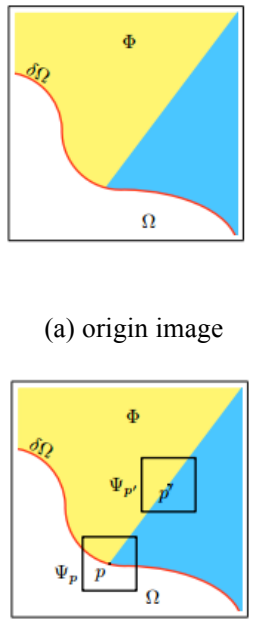

(c) use the similar patch to inpaint (a) origin image

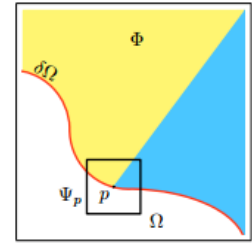

(b) computing patch priorities

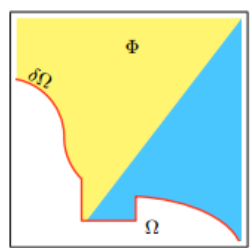

(d) complete one inpainting
Fig.1 the theory of exemplar-based method

Then the detail of the method contains three parts: in the image I, the $\Phi$ is the source region, the region $\Omega$ is destroyed by some reasons, and the $\partial \Omega$ is the edge between source region and destroyed region (Fig.1(a)). 
Firstly we need to compute the patch priorities to decide the inpainting sequence (Fig.1(b)). Secondly, by searching the most similar patch, we propagate the texture and structure information from the perfect part to the destroyed part(Fig.1(c)). Thirdly, update the confidence values and go to the next inpainting. At last, we loop these processing until the whole image is inpainted(Fig.1(d)).

\subsection{Computing patch priorities}

The proposed algorithm defines the inpainting sequence by the patch priorities, and this parameter is depend on two terms: (i)if the center pixel of the patch is located on a strong edge of the image, then it has a higher inpainting priorities;(ii)if the patch has more pixels that the gray values are known, then it has a higher inpainting priorities. The Fig. 2 shows the theory.

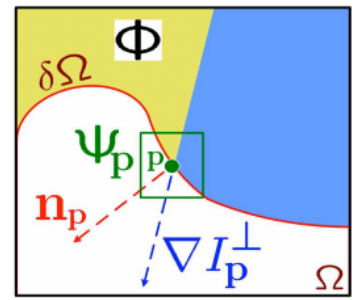

Fig.2 Computing the patch priorities

So we define the priorities $P(p)$ as the product of two terms:

$$
P(p)=C(p) * D(p)
$$

The $\mathrm{C}(\mathrm{p})$ which we call the confidence term means that if the patch has more known pixels, then it makes a large influence in the inpainting processing. It is defined as the follows'

$$
C(p)=\frac{\sum_{q \in \Psi_{p} \cap \bar{\Omega}} C(q)}{\left|\Psi_{p}\right|}
$$

In it, the function $C(p)$ is set to $C(p)=0 \quad \forall p \in \Omega$, and $\mathrm{C}(\mathrm{p})=1 \quad \forall p \in I-\Omega,\left|\Psi_{p}\right|$ means the number of the pixels in the patch.

On the other hand, the $\mathrm{D}(\mathrm{p})$ which we call the data term means that if the patch is located in the edge of the image, then it makes a large influence in the inpainting processing.

$$
D(p)=\frac{\left|\nabla I_{p}^{\perp} \bullet n_{p}\right|}{\alpha}
$$

In it, $\nabla I_{p}^{\perp}$ means the gradient of the pixel $p$, the $n_{p}$ means the unit vector orthogonal to the edge $\partial \Omega$ in the pixel p, the $\alpha$ means the gray scale of the image.

\subsection{Propagating the texture and structure}

We propagate image texture by direct sampling of source region, we search in the source region for that patch which is most similar to the patch $p$. this processing can be defined as the follows:

$$
\Psi_{\bar{q}}=\arg \min _{\Psi_{q} \in \Phi} d\left(\Psi_{\bar{p}}, \Psi_{q}\right)
$$

Here the $d(x, y)$ means the distance function of two patches, detail to say it is the sum of squared differences (SSD) of the already known pixels in the two patches. This time $d(x, y)$ we just use the Euclidean distance.

Having found the source exemplar patch $q$, each destroyed pixel in patch $\mathrm{p}$ need to be inpainted, it is just copied from its corresponding position inside patch $q$.

\subsection{Update the confidence values}

After the patch $p$ has been completed inpainted by the patch $q$, we need to update the confidence $C(p)$ in the area so that we can go into the further inpainting processing.

This simple update rule allows us to measure the relative confidence of patches on the fill front, without image specific parameters. As filling proceeds, confidence values decay, indicating that we are less sure of the color values of the pixels near the target region.

\section{Improvement}

\subsection{Adaptive sample size algorithm}

In the inpainting processing, the size of the sample patch always plays an important role in the inpainting processing. A larger size patch may remain the detail texture, but it would cause a low matching rate. While a smaller sample may spread the information of the edge, but it lost the detail texture. On another side, the size of the sample patch also makes an influence in the calculation time, the larger sample would reduce the time in selecting the sample while improving the time in computing the difference between patches. While the smaller sample would reduce the time spent in computing the difference between patches but improving the time spent in selecting the sample. So how to decide the size of the sample is very important. Here we employ an adaptive function to decide the size of the sample.

Firstly we introduce a concept of patch sparsity $(S(p))$.it shows the and $S(p)$ is define as: 


$$
S(p)=\sqrt{\left[\sum_{q \in N_{s}(p)} \omega_{p, q}^{2}\right] \cdot \frac{\left|N_{s}(p)\right|}{|N(p)|}}
$$

In it, $N(\mathrm{p})$ is number of the pixels in the neighbor region, $N_{s}(\mathrm{p})$ is the number of the known pixels in the neighbor region. $\omega_{p, q}$ means the similarity between the patch $\Psi_{p}$ and patch $\Psi_{q}$, and it is defined as:

$$
\omega_{p, q}=\frac{1}{Z(\mathrm{p})} \exp \left(-\frac{d\left(\Psi_{p}, \Psi_{q}\right)}{25}\right)
$$

The $Z(p)$ is a normalized constant which makes the $\sum_{q \in N_{s}(p)} \omega_{p, q}=1$

The value of the $S(p)$ represents the location of the patch, the patch locates in the edge region when the value is very large it locates in the texture region when the value is smaller and it locates in the stable region when the value is the smallest.

And then we set the sample size $\mathrm{w}$ as

$$
w=\left\{\begin{array}{l}
0.75 * w_{0} \quad S(p) \geq \lambda_{1} \bullet\left(p_{\max }-p_{\min }\right)+p_{\min } \\
w_{0} \quad \lambda_{2} \bullet\left(p_{\max }-p_{\min }\right)+p_{\min } \leq S(p) \leq \lambda_{1} \bullet\left(p_{\max }-p_{\min }\right)+p_{\min } \\
1.25 * w_{0} \quad S(p) \leq \lambda_{2} \bullet\left(p_{\max }-p_{\min }\right)+p_{\min }
\end{array}\right.
$$

w0 is the standard sample size, it depends on the image size and the destroyed region size, here we use the fortieth of the destroyed region as it. pmax and pmin are the maximum and minimum of all the patch sparsity. $\lambda_{1}$ and $\lambda_{2}$ threshold set by the experience, here we set $\lambda_{1}$ equals 0.55 and $\lambda_{2}$ equals 0.15 .

\subsection{Candidate patch system}

Although the traditional method can contribute a good result while inpainting the nature image, if it is used to inpaint the image with more complex texture ,then it would come out many errors. After many times testing I find the reason is the patch selecting system. The traditional method uses template matching method to select the inpainting patches. Sometimes the sample has several similar matching patches, the global difference between these patches are very small, but the differences are just focused in a small region, then it is hard for the system to select the correct patches. So this paper proposes a new patch selecting method. Firstly it selects not only the most similar patch, but also several candidate patches. Secondly, we select the three most similar patches, and calculation the difference among them, if it is very small, it means that the location of these patches are nearly the same place, so we can just select the most similar patch to inpaint. If one of the differences is larger than a threshold, then it expands the patch size to make sure selecting the correct patch. Fig.3 shows an example of the candidate patch system, in (a), the right patches are the three most similar patches to the sample patch. For the traditional method, it may select the top patch because it has the minimal difference. But if we expand the sample patch and the candidate patches, we can find that the third patch is more significant to the sample patch.

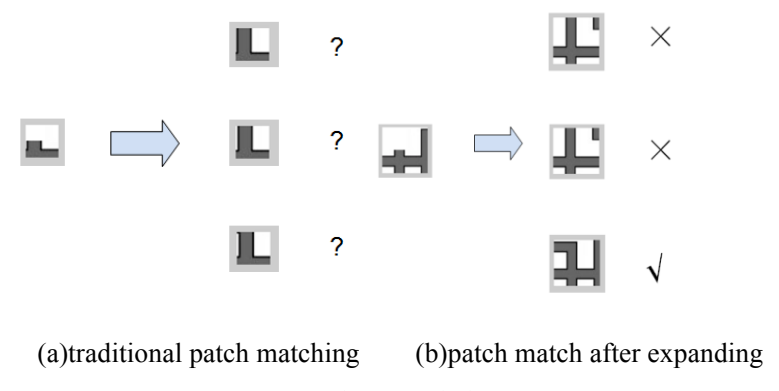

Fig. 3 The candidate system

From the result we can find that the improvement makes a contribution on improving the correct matching rate.

\section{Experiment and result}

Here we apply our algorithm to a set of images, ranging from simple graphic to nature images that including complex textures. Where possible, we make side-by-side comparisons to previously proposed methods. In other cases, we hope the reader will refer to the original source of our test images and compare these results with the results with the results of earlier work.

In all of the experiments, the patch size is set as the fortieth of the destroyed region, and the source region has been set to be the remaining of the destroyed image. All experiments are made by the tool matlab 7.10.0 and run on a $2.8 \mathrm{GHz}$ Pentium CPU G640 with 4GB of RAM.

Firstly, we perform the proposed method to inpaint a simple graphic like Figure 4 show us.

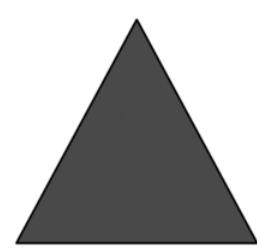

(a) origin image

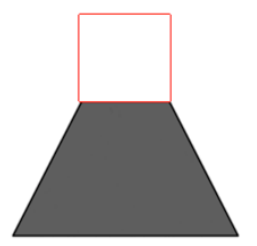

(b) destroyed image 


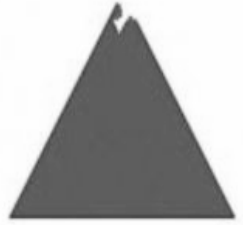

(c) traditional result

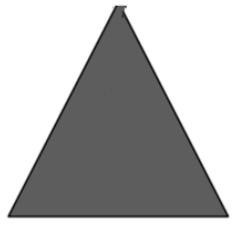

(d)proposed result
Fig.4 Inpainting result on a simple graphic

By some reasons, origin image Fig.4 (a) is destroyed (b). To inpaint the destroyed image, compared with the traditional exemplar-method result(c), the proposed method(d) adaptively changed the sample size to inpaint and we can find that the inpainting process search the more significant patches until to the top of the graphic. Because the top part we can not find a similar patch to inpaint it and at last it just select a relative simple patch to inpaint. Due to the disadvantage of exemplar-based method, we can not perfectly inpaint the corner, but the proposed method tried its best to reduce the error region.

Secondly, Fig.5 shows the experiment on a complex graphic. For the origin image Fig.5(a), to inpaint the destroyed one(b), for the traditional method(c), after the inpainting processing entering into the inside region, it may select a similar but not correct patch(like the chapter 3.2 shows us) because the difference between them is also very small and at last it gives a result without logic, but the proposed method(d) can overcome the disadvantage by using the candidate patch system.

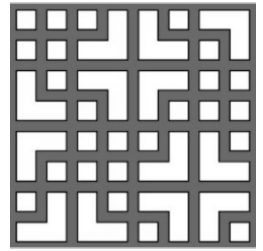

(a) origin image

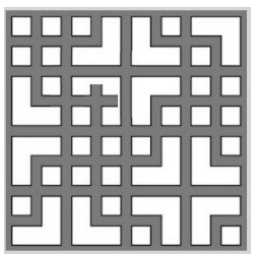

(c) traditional result

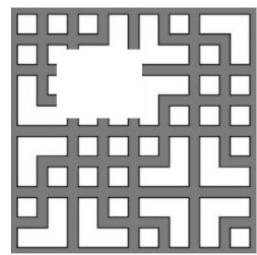

(b) destroyed image

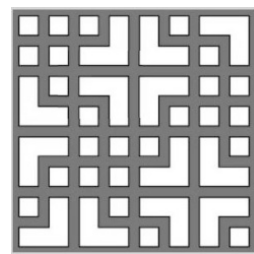

(d)proposed result
Fig.5 Inpainting result on a complex graphic

What's more, we try to inpaint a scenery image (Fig.6):

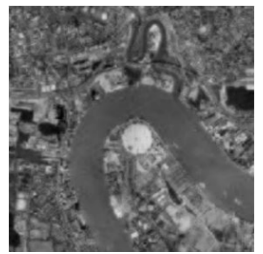

(a) origin image

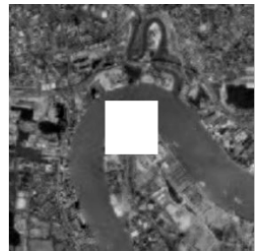

(b) destroyed image

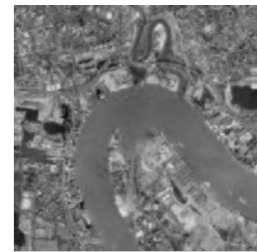

(c) pixel level result

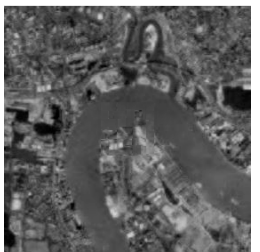

(d) exemplar-based result

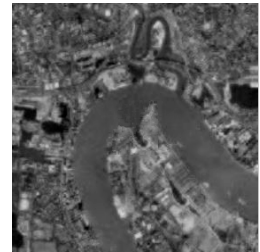

(e) proposed result
Fig.6 Inpainting result on a natural image

For the origin image Fig.6(a) To inpaint the destroyed version(b), (c) inpainted the image on pixel level so the result is very fuzzy around the edge, and when inpainting entering into the inside, it even gives none of useful information only maintain the integrity of the original image. Traditional method(d) inpainted the image on patch level so it can remain some texture information inside, but the result is not very good. The proposed method(e) has a better result in some place, especially in the connection part between destroyed part and the source part. It gives a quite different visual effect. The detail result is shown in Fig.7 (the red line shows the destroyed edge.), from these result we can see the proposed method has two advantages. Firstly, it has a better connection to the remaining region. Secondly, compared with the others two results, the proposed method also has a better effect on the connection of different patches. As I think, the differences are contributed by the improvement of the patch selection. The improvement enhances the edge effect of the image and has a better connection effect between different patches, so at last the result has a strong edge and a smooth inpainting content after the processing.

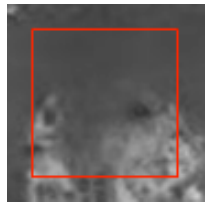

(a) pixel level result

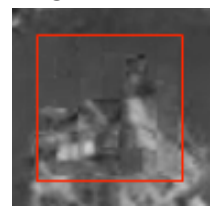

(b) exemplar-based result

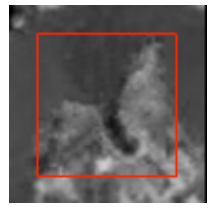

(c) proposed result
Fig.7 Detail result of Fig.6

\section{Conclusion and future work}

In this paper, two improvements are proposed to improve the correct matching rate, it can overcome the disadvantage made by the traditional exemplar-based method. The first one is using an adaptive sample, it divides the template matching process into three types and adaptively changing the sample size according to the sample patch type, which is different from the traditional method; The second one is employing a candidate patch system. Compared with the traditional method, it firstly selects a series of similar patches, not only the most similar 
patch, if the differences between these candidate patches are larger than a threshold, then spread the calculation region for further calculation the difference between the sample patch and the candidate patches. At last it selects the more significant patch. By the result, we can see it not only catches the correct patches but also in a way improves the pertinence to different region in the inpainting processing.

Now in the template matching part of proposed method, it just uses the grey value information. In the future, we would try to add feature extraction phase to add the rotation invariance in it and then it can overcome the disadvantage caused in Figure 4.

\section{References}

(1) M. Bertalmio, G. Sapiro, V. Caselles, and C. Ballester : "Imagein-painting," in Proc. SIGGRAPH, pp. 417-424.2000.

(2) M.Bertalmio, A. L. Bertozzi, and G. Sapiro : "Navier-Strokes, fluid dynamics, and image and video in-painting," in Proc. IEEE Computer Society Conf. Computer Vision and Pattern Recognition, pp.417-424. 2001.

(3) $\mathrm{H} \mathrm{Lu}, \mathrm{L}$ Zhang, and S Serikawa : "Maximum local energy: An effective approach for multisensor image fusion in beyond wavelet transform domain," in Computers \& Mathematics with Applications, vol.64, no.5, pp.996-1003, 2012.

(4) S.serikawa and $\mathrm{H} \mathrm{Lu}$ : "Underwater image dehazing using joint trilateral filter" in Computers \&Electrical Engineering vol.40,no.1,pp.41-50, 2014.

(5) M. Afonso , J. Bioucas-Dias and M. Figueiredo : "An augmented Lagrangian approach to the constrained optimization formulation of imaging inverse problems", IEEE Trans. Image Process., vol. 20, no. 3, pp.681 -695.2011

(6) Zhu Yong, Wang Gui, Han Zhike : “A New Oriented-Diffusion Image Inpainting Framework for Striped Texture Images". Published in: International Forum on Information Technology and Applications, pp.79-84. 2009

(7) Chuang Zhu, Huizhu Jia, Meng Li, Xiaofeng Huang : "Highly Efficient Local Non-Texture Image Inpainting Based on Partial Differential Equation". Published in:International IEEE 17th Conference on Computational Science and Engineering (CSE), pp.803-807, 2014.
(8) A. Telea : "An image in-painting technique based on the fast marching method," in Journal of Graphics Tools, vol 9,no. 1,pp.23-34, 2004.

(9) Y. Yang, X. Juan : "An improved image in-painting algorithm based on fast marching method," in Journal of Xi'an University of Technology, vol.25, no.2, pp.129-134,2009.

(10) Qian Fan, Xuelong Hu, Lifeng Zhang : "Image inpainting based FMM algorithm by a direction selection method", Proc. of ICIAE2014, pp.12-15, 2014.

(11) A. Criminisi, P. Perez, and K. Toyama : "Region filting and object removal by examplar-based image inpainting," in IEEE Trans. Image Process.,vol. 33,pp. 1200-1212,2004.

(12) A. Efros and T. Leung, "Texture synthesis by non-parametric sampling," in Proceedings of International Conference on Computer Vision, pp. 1033-1038, 1999.

(13) M. Bertalmio, L. Vese, G. Sapiro, and S. Osher : "Simultaneous structure and texture image in-painting," IEEE Trans. Image Process., vol. 12, pp. 882-889, 2003.

(14) D. Zoran and Y. Weiss : "From learning models of natural image patchesto whole image restoration", Proc. Int. Conf.Comput. Vis. pp.479 -486. 2011

(15) A. Bugeau , M. BertalmÃo , V. Caselles and G. Sapirob : "A Comprehensive Framework for Image Inpainting", IEEE Trans. Image Processing, vol. 19, no. 10 , pp. 2634-2645, 2010

(16) Shu-Chiang Chung,Ta-Wen Kuan, Chuan-Pin $\mathrm{Lu}, \mathrm{Hsin}-\mathrm{Yi}$ Lin : "A new approach of image inpainting based on PSO algorithm", Published in International Conference on Orange Technologies (ICOT), pp. 205-209, 2013

(17) Li Zhi dan,He Hongjie, Yin Zhongke, Chen Fan, Renqing Nuobu : "Adaptive Image Inpainting Algorithm Based on Patch Structure Sparsity." Published in the ACTA ELECTRONICA SINICA, pp.549-554,2013

(18) R.Martinez-Noriega, A.Roumy, G.Blanchard : "Exemplar-based image inpainting:fast priority and coherent nearest neighbor search." in IEEE International Workshop on Machine Learning for Signal Processing,pp.1-6,2012 